\title{
Egr-1 and RNA POL II facilitate glioma cell GDNF transcription induced by histone hyperacetylation in promoter II
}

\author{
Bao-Le Zhang ${ }^{1}$, Ting-Wen Guo ${ }^{1}$, Le-Le Gao ${ }^{1}$, Guang-Quan ${ }^{1}{ }^{1}$, Xiao-He Gu${ }^{1}$, Yu-Qi \\ Shao', Rui-Qin Yao ${ }^{1}$ and Dian-Shuai Gao ${ }^{1}$ \\ ${ }^{1}$ Department of Neurobiology and Anatomy, Xuzhou Key Laboratory of Neurobiology, Jiangsu Key Laboratory of New Drug \\ Research and Clinical Pharmacy, Xuzhou Medical University, Xuzhou 221004, Jiangsu, China
}

Correspondence to: Dian-Shuai Gao, email: gds@xzhmu.edu.cn

Keywords: Egr-1, GDNF, RNA POL II, histone acetylation, glioma

Received: December 27, $2016 \quad$ Accepted: January 25, $2017 \quad$ Published: February 06, 2017

Copyright: Zhang et al. This is an open-access article distributed under the terms of the Creative Commons Attribution License 3.0 (CC BY 3.0), which permits unrestricted use, distribution, and reproduction in any medium, provided the original author and source are credited.

\section{ABSTRACT}

The specific mechanisms for epigenetic regulation of gene transcription remain to be elucidated. We previously demonstrated that hyperacetylation of histone H3K9 in promoter II of glioma cells promotes high transcription of the glial cell line-derived neurotrophic factor (GDNF) gene. This hyperacetylation significantly enhanced Egr-1 binding and increased the recruitment of RNA polymerase II (RNA POL II) to that region $(P<0.05)$. Egr-1 expression was abnormally increased in $C 6$ glioma cells. Further overexpression of Egr-1 significantly increased Egr-1 binding to GDNF promoter II, while increasing RNA POL II recruitment, thus increasing GDNF transcription $(P<0.01)$. When the acetylation of H3K9 in the Egr-1 binding site was significantly reduced by the histone acetyltransferase (HAT) inhibitor curcumin, binding of Egr-1 to GDNF promoter II, RNA POL II recruitment, and GDNF mRNA expression were significantly downregulated $(P<0.01)$. Moreover, curcumin attenuated the effects of Egr-1 overexpression on Egr-1 binding, RNA POL II recruitment, and GDNF transcription $(P<0.01)$. Egr-1 and RNA POL II co-existed in the nucleus of $\mathrm{C} 6$ glioma cells, with overlapping regions, but they were not bound to each other. In conclusion, highly expressed Egr-1 may be involved in the recruitment of RNA POL II in GDNF promoter II in a non-binding manner, and thereby involved in regulating GDNF transcription in high-grade glioma cells. This regulation is dependent on histone hyperacetylation in GDNF promoter II.

\section{INTRODUCTION}

Glial cell line-derived neurotrophic factor (GDNF) is a member of the transforming growth factor (TGF- $\beta$ ) superfamily and an important neurotrophic factor. It was initially cloned from the rat B49 glial cell line [1]. In human cells, GDNF is a single-copy gene with two promoters and six exons located on chromosome 5 at p12-p13.1 $[2,3]$. As it is a powerful factor promoting glioma cell proliferation and migration, GDNF is closely related to glioma development [4-8]. Its transcription is significantly higher in glioma cells than in normal glial cells $[9,10]$. We previously reported that abnormal highlevel GDNF transcription in glioma cells was related to histone hyperacetylation in its promoter II rather than gene mutation [11]. A subsequent study showed three consecutive and highly conserved binding sites for early growth response protein-1 (Egr-1) were located -186 bp upstream of the transcription initiation site in rat $G D N F$ promoter II [12]. Histone hyperacetylation at these sites facilitates high-level GDNF transcription in C6 glioma cells [13], but the specific mechanism has not yet been elucidated.

The transcription factor Egr-1 has been shown to regulate cell growth, differentiation, and tumor progression [14-16]. It is highly expressed in tumor tissues such as liver and prostate cancer tissues $[17,18]$. Moreover, Egr1 overexpression has been shown to significantly promote the infiltration and migration of high-grade glioma cells $[19,20]$, which is consistent with the biological effect induced by the high-level GDNF transcription $[4,7]$. We therefore hypothesized that Egr-1 expression might also be abnormally increased in high-grade glioma cells and that this transcription factor could mediate histone hyperacetylation-induced high-level GDNF transcription. In addition, bioinformatics analysis revealed that the 
upstream sequence of the GDNF gene transcription initiation site was rich in $\mathrm{CG}$ bases. Moreover, no typical TATA-box motif was found from -25 to $-30 \mathrm{bp}$, which is to say GDNF promoter II region is TATA-less. CG-rich TATAless promoters are thought to recruit RNA polymerase II (RNA POL II) by increasing their binding to gene-specific transcription factors, thereby initiating or promoting gene transcription [21]. Three consecutive and conserved Egr-1 binding sites are located in the $\mathrm{CG}$-rich region upstream of the GDNF promoter II [13]. Therefore, we further hypothesized that Egr-1 might be involved in histone hyperacetylation-mediated GDNF transcription in highgrade glioma cells by increasing RNA POL II recruitment to the Egr-1 binding sites of GDNF promoter II.

We examined acetylation of histone H3 Lys9 (H3K9) in the Egr-1 binding sites of GDNF promoter II, as well as their binding to Egr-1 and RNA POL II, in normal and diseased human tissue by chromatin immunoprecipitation (ChIP). Subsequently, the expression of Egr-1 in human high-grade and low-grade glioma tissues, normal brain tissue, and rat C6 astroglioma cells and normal astrocytes were measured by real-time polymerase chain reaction (PCR) and western blot. Next, the effects of Egr-1 overexpression on Egr-1 binding to GDNF promoter II, RNA POL II recruitment, and GDNF transcription in C6 glioma cells were determined by gene overexpression, ChIP, and real-time PCR. The effects of Egr-1 overexpression on these outcomes were elucidated using a C6 glioma cell model with decreased histone acetylation in GDNF promoter II following treatment with the histone acetyltransferase (HAT) inhibitor curcumin [22-24]. Finally, the presence and mutual binding of RNA POL II and Egr-1 in the nuclei of C6 astroglioma cells were assessed by immunofluorescence and co-immunoprecipitation (co-IP). Our findings provide new clues to the mechanism underlying abnormally high transcription of GDNF in glioma cells.

\section{RESULTS}

\section{Increased $\mathrm{H} 3 \mathrm{~K} 9$ acetylation in Egr-1 binding sites of $G D N F$ promoter II in high-grade glioma tissue}

Three consecutive and conserved Egr-1 binding sites were present -32 bp upstream of the transcription initiation site in human GDNF promoter II (Figure 1A). H3K9 acetylation in the Egr-1 binding sites (on-target region $-98 /+56 \mathrm{bp})$ and off-target region (-864/-793 bp) of GDNF promoter II was measured in high-grade and low-grade glioma tissues and normal brain tissue using ChIP-PCR. The primer positions are shown in Figure 1A. The histone H3K9 in the Egr-1 binding sites of GDNF promoter II was acetylated to varying degrees (Figure 1B). Acetylation was significantly higher in the Egr-1 on-target region of $G D N F$ promoter II $(P<0.01)$ and the off-target region $(P<0.05)$ in high-grade glioma tissue compared with normal brain tissue. Moreover, the degree of acetylation was significantly higher in the Egr-1 ontarget region than in the off-target region $(P<0.01)$. This is consistent with our previous report of a difference in H3K9 acetylation in GDNF promoter II of rat C6 glioma cells [13]. By contrast, there was no significant difference in $\mathrm{H} 3 \mathrm{~K} 9$ acetylation in the Egr-1 on- and off-target regions in low-grade glioma tissue compared with normal brain tissue $(P>0.05$, Figure $1 \mathrm{C})$.

\section{Increased Egr-1 binding to GDNF promoter II in high-grade glioma tissue}

Egr-1 binding to GDNF promoter II was measured by ChIP-PCR. The results showed that Egr-1 could bind between -98 and +56 of $G D N F$ promoter II in all three types of tissues but not to the off-target region (between -864 and -793). This demonstrated the specific binding of Egr-1 to the Egr-1 binding sites of GDNF promoter II (Figure 2A). Compared with normal brain tissue, Egr-1 binding was very significantly increased in high-grade glioma tissue $(P<0.01)$. This is consistent with the difference in $\mathrm{H} 3 \mathrm{~K} 9$ acetylation in $G D N F$ promoter II of rat C6 glioma cells we previously reported [13]. There was no significant difference between low-grade glioma tissue and normal brain tissue $(P>0.05$, Figure $2 \mathrm{~B})$.

\section{Increased recruitment of RNA POL II to the Egr-1 binding sites of $G D N F$ promoter II in high-grade glioma tissue and cells}

RNA POL II binding to the Egr-1 binding sites of $G D N F$ promoter II was measured by ChIP-PCR to assess the recruitment of RNA POL II in the Egr-1 binding sites of GDNF promoter II in different grades of glioma. The results showed that RNA POL II could bind to different extents in all three types of tissues (Figure 3A). Compared with normal brain tissue, RNA POL II recruitment to the Egr-1 binding sites was significantly increased in highgrade glioma tissue $(P<0.05)$. In contrast, there was no significant difference between low-grade glioma tissue and normal brain tissue $(P>0.05$, Figure $3 \mathrm{~B})$. To further determine whether this phenomenon occurs in high-grade glioma cells, RNA POL II recruitment to Egr-1 binding sites (between -375 and -93 ) of rat GDNF promoter II was measured in rat C6 astroglioma cells and normal astrocytes using ChIP-PCR. The results showed that RNA POL II recruitment was also significantly increased in $\mathrm{C6}$ astroglioma cells $(P<0.01$, Figure $3 \mathrm{C}$ and $3 \mathrm{D})$.

\section{Increased Egr-1 expression in high-grade glioma tissue and cells}

Egr-1 expression was measured in high-grade and low-grade glioma tissues and normal brain tissue using real-time PCR and western blot. The results showed that 
mRNA and protein expression of Egr-1 in high-grade glioma tissue were significantly higher than that in normal brain tissue $(P<0.01)$. In contrast, there was no significant difference between low-grade glioma and normal brain tissues $(P>0.05$, Figure 4A and 4B). To further determine whether this phenomenon is also present in high-grade glioma cells, Egr-1 expression was measured in rat $\mathrm{C} 6$ astroglioma cells and normal astrocytes using real-time PCR and western blot. The results showed that the mRNA and protein expressions of Egr-1 in C6 glioma cells were also significantly higher than that in normal astrocytes $(P<0.01$, Figure 4C and 4D).

A Human GDNF gene

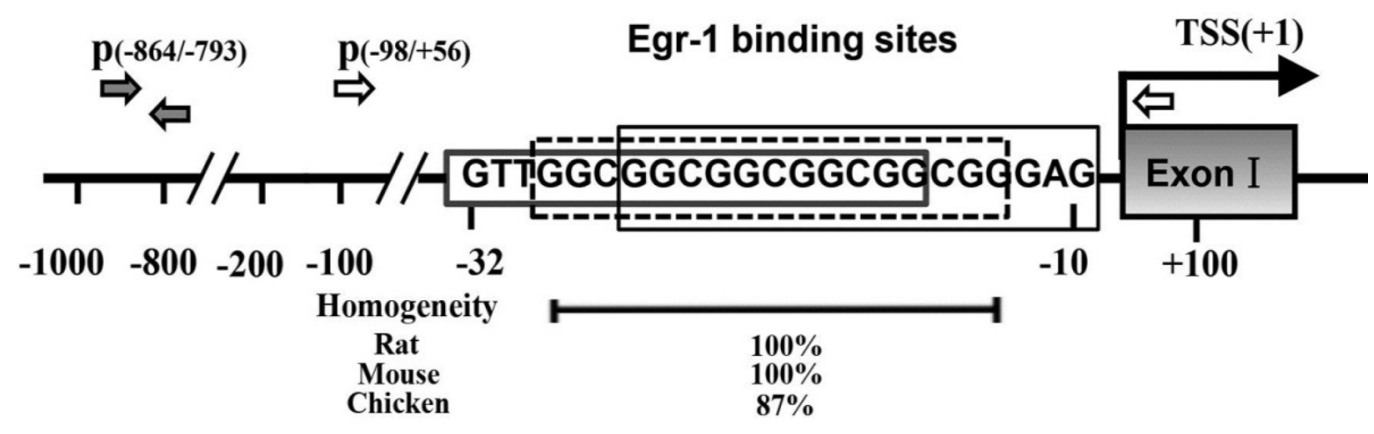

B
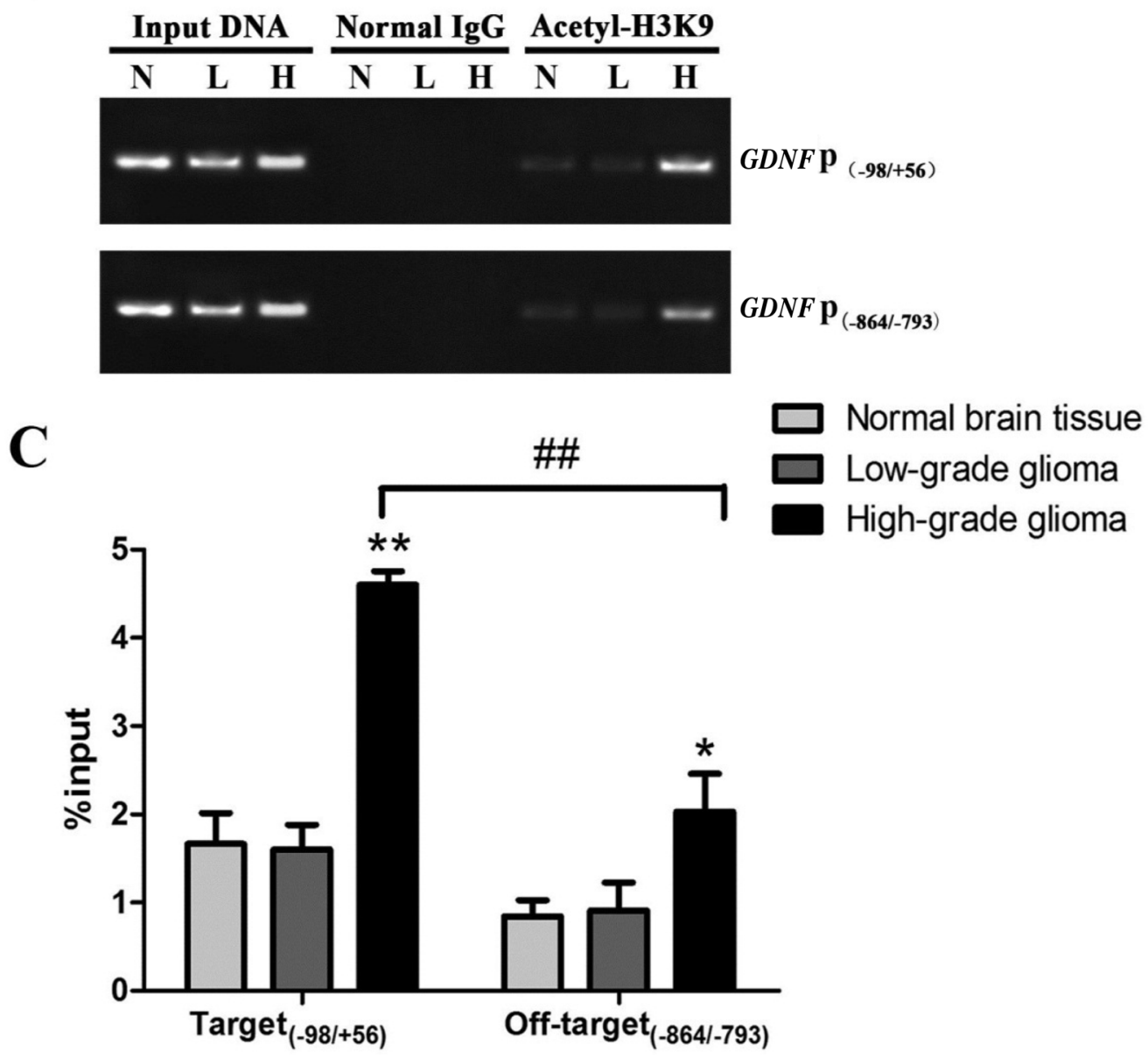

Figure 1: H3K9 acetylation of GDNF gene promoter region II in human high-grade glioma tissue (H), low-grade glioma tissue (L), and normal brain tissue (N). (A) Schematic showing the Egr-1 binding site in human GDNF gene promoter region II. The black and white arrows indicate the ChIP-PCR primer positions. The transcription start site (TSS) of the promoter II-derived transcript is +1. Phylogenetic conservation values are indicated. (B) ChIP analyses with anti-acetyl-histone H3 (Lys9) antibody or normal rabbit IgG (negative control). Immunoprecipitated and input DNA were assayed using PCR with specific primers $(\mathrm{P}(-98 /+56), \mathrm{P}(-864 /-793))$. (C) The ChIP DNA value was standardized with input DNA, and the data are the percentages of input chromatin. ${ }^{*} P<0.05,{ }^{* *} P<0.01,{ }^{\# \#} P<0.01$. 


\section{Egr-1 overexpression significantly increased RNA POL II recruitment to GDNF promoter II and $G D N F$ transcription in $\mathrm{C} 6$ glioma cells}

To investigate the relationship of Egr-1 overexpression with RNA POL II recruitment to GDNF promoter II and GDNF transcription in high-grade glioma cells, C6 astroglioma cells were infected with 100 MOI of lentivirus Egr1-OE. The infection rate was then determined $72 \mathrm{~h}$ later with fluorescence microscopy. Egr- 1 overexpression was examined by real-time PCR and western blot. The effects of Egr-1 overexpression on the binding of Egr-1 and RNA POL II to GDNF promoter II and $G D N F$ transcription were examined by ChIP-PCR and real-time PCR in C6 astroglioma cells. The results showed that the infection efficiency of lentivirus Egrl-OE was > $90 \%$, and the cells were in good condition after infection (Figure 5A and 5B). Both mRNA (Figure 5C) and protein (Figure 5D) expression of Egr-1 were significantly increased $(P<0.01)$ after infection, indicating the successful establishment of a C6 glioma cell model with overexpressed Egr-1. Furthermore, Egr-1 overexpression in C6 astroglioma cells significantly increased both binding of Egr-1 to the Egr-1 binding sites of GDNF promoter II $(P<0.05$, Figure 5E) and recruitment of RNA POL II in that region (Figure 5F). GDNF transcription was also significantly enhanced $(P<0.01$, Figure $5 \mathrm{G})$. Notably, the results showed that Egr-1 protein expression had the most significant positive correlation with GDNF transcription $(\mathrm{r}=0.974, P<0.01)$.

\section{Histone hypoacetylation in the Egr-1 binding sites of $G D N F$ promoter II inhibited $G D N F$ transcription induced by Egr-1 overexpression in C6 glioma cells}

To determine whether histone hyperacetylation in the Egr-1 binding sites of GDNF promoter II is necessary for Egr-1 to regulate enhanced GDNF transcription in glioma cells, a C6 glioma cell model with histone hypoacetylation in the Egr-1 binding sites of GDNF promoter II was established by treatment with curcumin (Figure 6A). The effects of Egr-1 overexpression on histone acetylation, Egr-1 binding to GDNF promoter II, RNA POL II recruitment, and GDNF transcription in curcumin-treated $\mathrm{C} 6$ glioma cells were examined by gene overexpression, ChIP, and real-time PCR studies. The results showed that $24 \mathrm{~h}$ after treatment with $50 \mu \mathrm{M}$ curcumin, H3K9 acetylation in the Egr-1 binding sites of $G D N F$ promoter II, Egr-1 binding to GDNF promoter II, RNA POL II recruitment in that region, and GDNF mRNA expression were significantly decreased compared with the DMSO treatment group (all $P<0.01$ ). Moreover, Egr-1 overexpression failed to significantly increase RNA POL II recruitment to the Egr-1 binding sites of GDNF promoter II or GDNF expression $(P>0.05)$. Conversely,
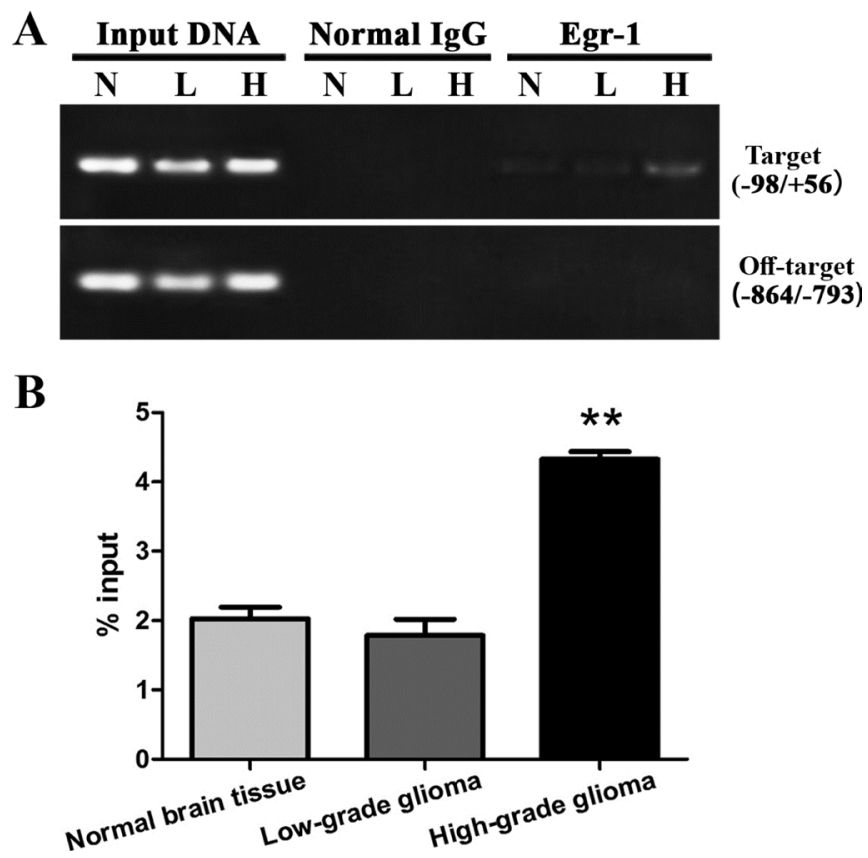

Figure 2: Egr-1 binding to GDNF gene promoter region II in human high-grade glioma tissue (H), low-grade glioma tissue (L), and normal brain tissue (N). (A) An anti-Egr-1 antibody or normal rabbit IgG was used for ChIP analysis following cross-linking and chromatin breakage. Specific primers for target $\mathrm{P}(-98 /+56)$ and off-target $\mathrm{P}(-864 /-793)$ areas and immunoprecipitated DNA and input DNA were detected with PCR. Specific primers for target area $\mathrm{P}(-98 /+56)$ amplified target fragments to variable degrees, but using the same ChIP DNA template, the primer for the off-target area P(-864/-793) could not produce target fragments with PCR amplification, while target fragments were procured using input DNA. (B) After real-time PCR, ChIP DNA values were standardized with input DNA, and data are the percentages of chromatin. ${ }^{* *} P<0.01$. 
we observed significantly increased $G D N F$ transcription in the DMSO treatment group $(P<0.001$, Figure 6$)$, which suggests that histone hyperacetylation is a prerequisite for highly expressed Egr-1 to regulate GDNF transcription. In addition, compared with the curcumin treatment group, Egr-1 overexpression after curcumin treatment did not significantly affect $\mathrm{H} 3 \mathrm{~K} 9$ acetylation in the Egr-1 binding sites of GDNF promoter II $(P>0.05)$, the Egr-1 binding to $G D N F$ promoter II $(P=0.061)$, and RNA POL II recruitment in that region $(P>0.05$, Figure 6A-6C).

\section{Co-existence but not binding of Egr-1 and RNA} POL II in high-grade glioma cell nuclei

To investigate the mode of action between Egr-1 and RNA POL II in high-grade glioma cells, the binding of Egr-1 to RNA POL II in C6 astroglioma cells was measured by double immunofluorescence and co-IP. The results showed that although both Egr-1 and RNA POL II were present in the nuclei of C6 glioma cells with local overlapping regions (Figure 7A), they were not bound to each other (Figure 7B-7C).
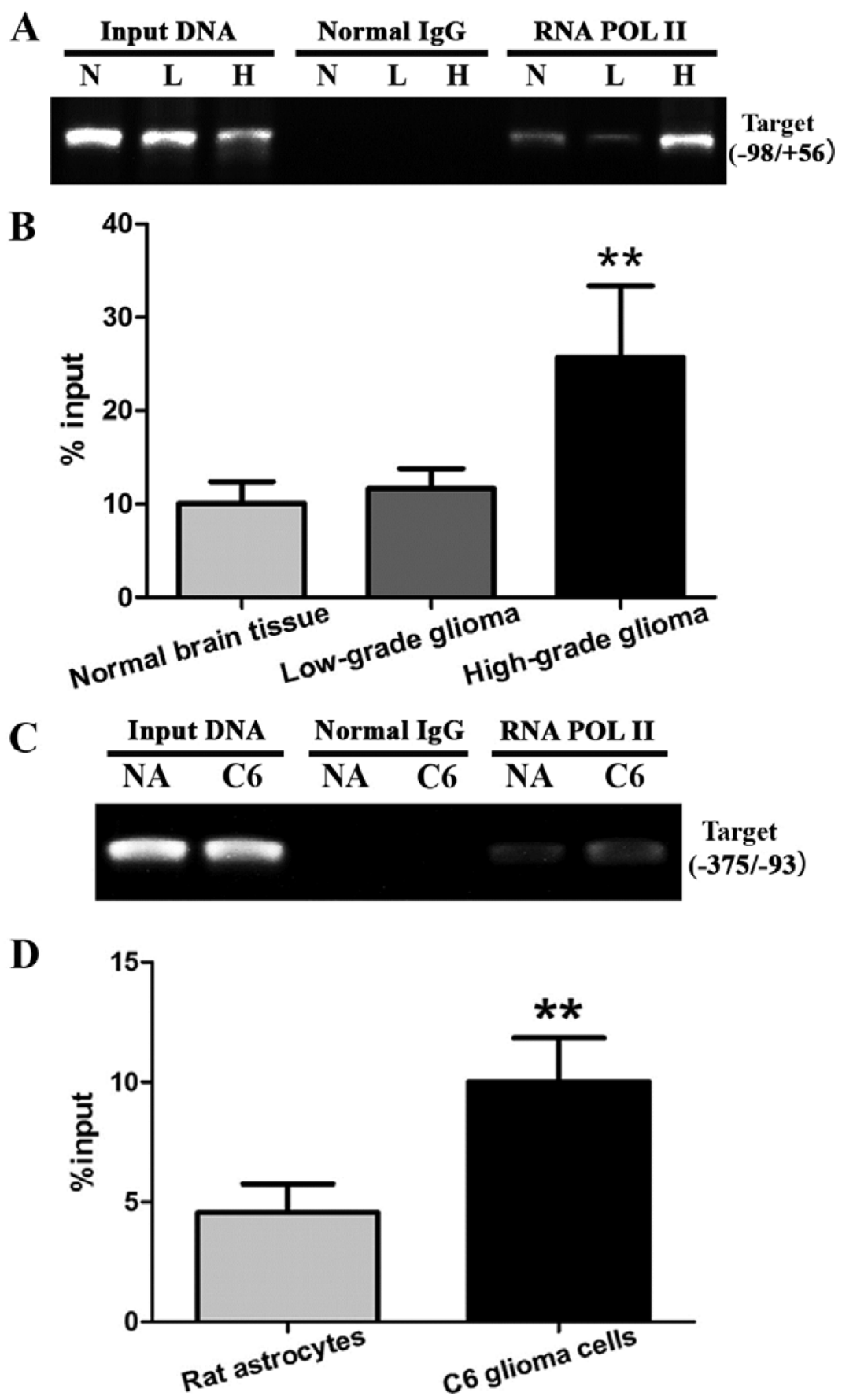

Figure 3: RNA POL II recruitment to Egr-1 binding sites of GDNF promoter II in glioma tissues and cells. (A, B) RNA POL II recruitment was measured in different grades of glioma tissues using ChIP-PCR. (C, D) Recruitment was measured in rat C6 astroglioma cells and normal astrocytes using ChIP-PCR. $* * P<0.01$. 


\section{DISCUSSION}

Egr-1 is a key transcription factor for $G D N F$ gene activation $[12,25,26]$ and is involved in histone hyperacetylation-mediated high-level GDNF transcription in glioma cells [13]. In this study, H3K9 acetylation in Egr-1 binding sites of GDNF promoter II, Egr-1 binding, and RNA POL II recruitment in that region were significantly increased in high-grade glioma tissue. It was therefore hypothesized that Egr-1 and RNA POL II might be synergistically involved in histone hyperacetylationmediated high-level GDNF transcription.
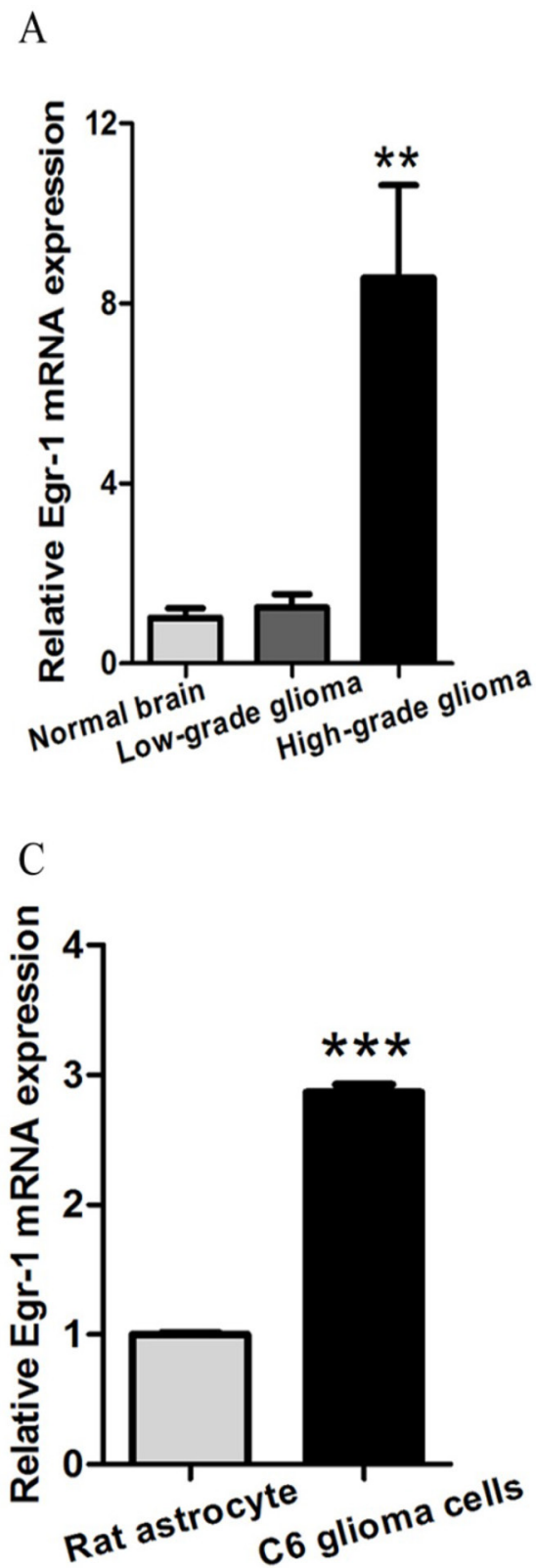

Egr-1 expression was measured in high- and lowgrade glioma and normal brain tissue, as well as rat C6 astroglioma cells and normal astrocytes. Compared with the control group, Egr-1 expression was significantly higher in high-grade glioma tissue and C6 astroglioma cells. This is consistent with the high expression of Egr-1 reported by Peng et al. $[17,18]$ in cancerous tissues such as liver cancer and prostate cancer. In addition, we previously found that $G D N F$ transcription was significantly decreased by the knockdown of Egr-1 in C6 glioma cells [13]. This indicates that highly expressed Egr-1 may be involved in regulating the high-level transcription of $G D N F$ in high-grade glioma cells.
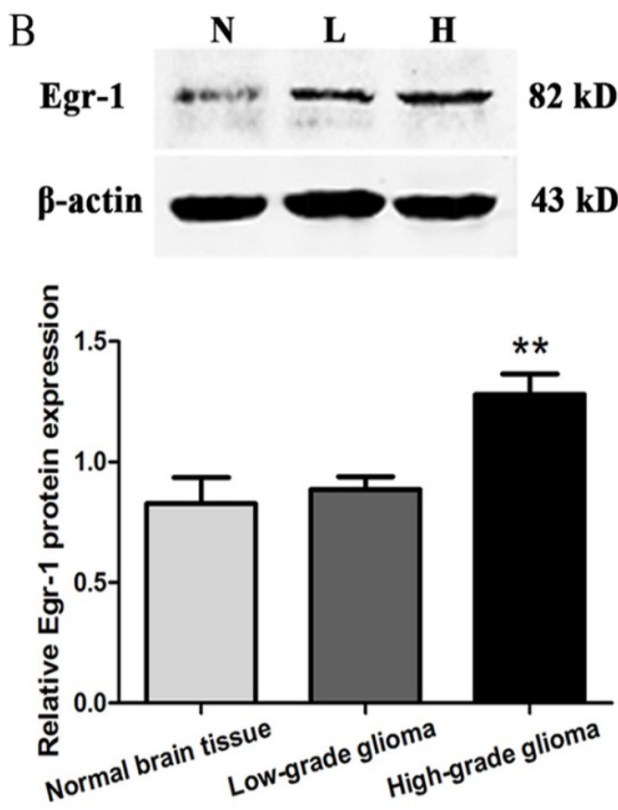

D NA C6
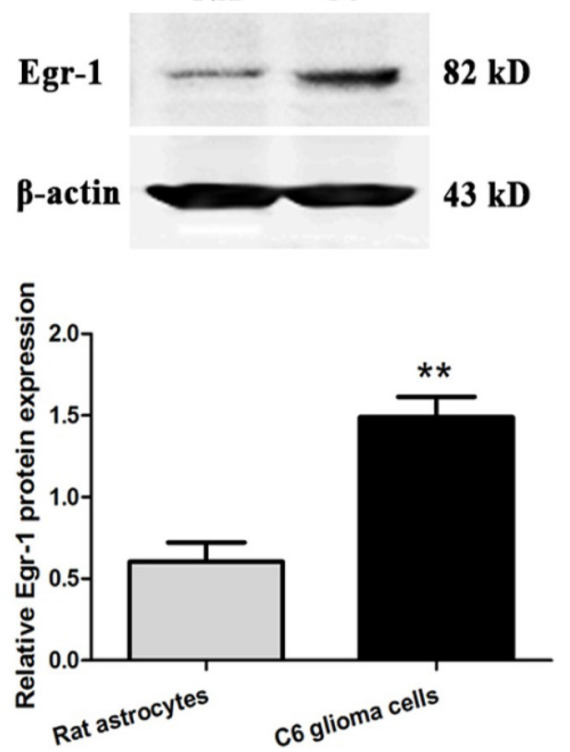

Figure 4: Egr-1 expression levels in glioma tissues and cells. (A, B) Relative expression of Egr-1 mRNA and protein in high-grade glioma tissue $(\mathrm{H})$, low-grade glioma tissue $(\mathrm{L})$ and normal brain tissue $(\mathrm{N})$. (C, D) Relative Egr-1 mRNA and protein expression in rat C6 astroglioma cells and rat normal astrocytes (NA). $\beta$-actin was used as the internal reference protein. ${ }^{* *} P<0.01,{ }^{* * *} P<0.001$. 
The effect of Egr-1 overexpression on GDNF transcription in glioma cells was examined to test this hypothesis. GDNF mRNA expression was significantly increased by Egr-1 overexpression in C6 glioma cells.

$\mathbf{A}$

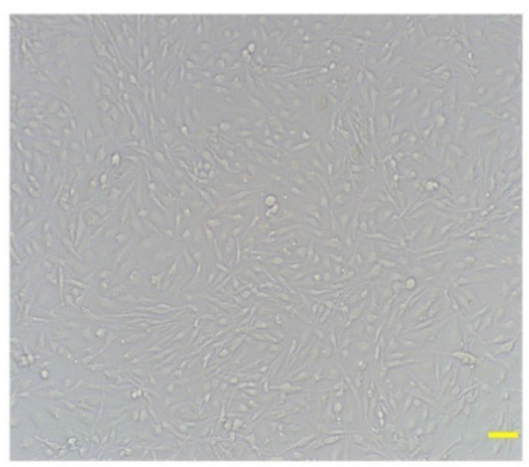

C
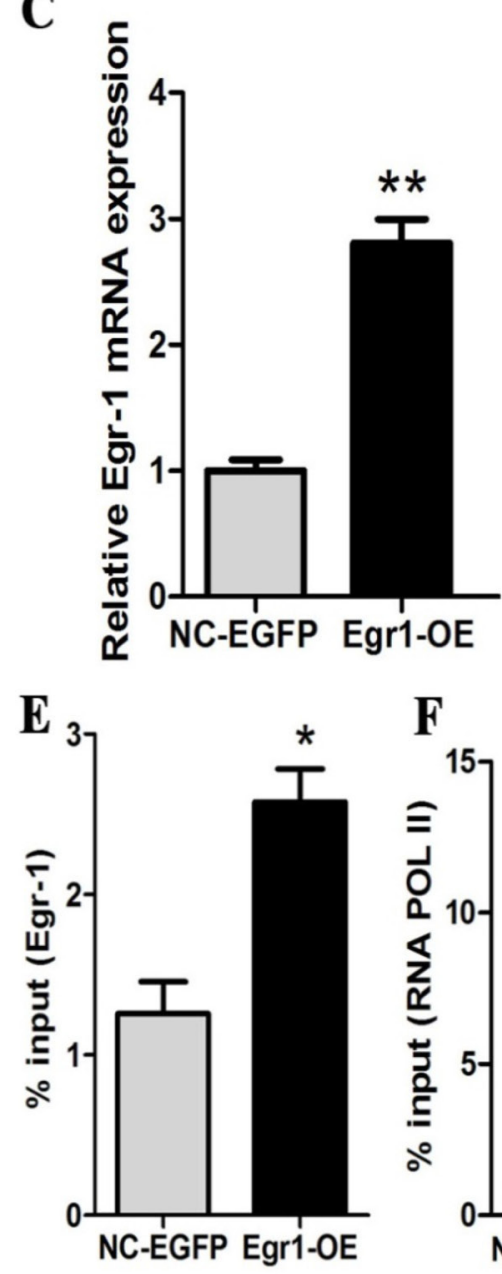

$\mathbf{F}$
Moreover, both binding of Egr-1 to GDNF promoter II and RNA POL II recruitment in that region were significantly increased under this condition. This is similar to the effect of histone hyperacetylation of the Egr-1 binding sites of

B

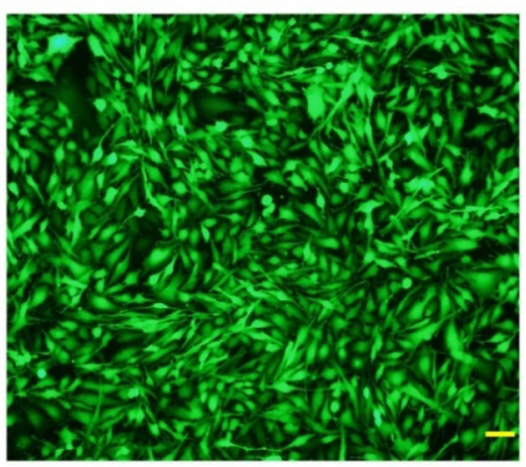

D
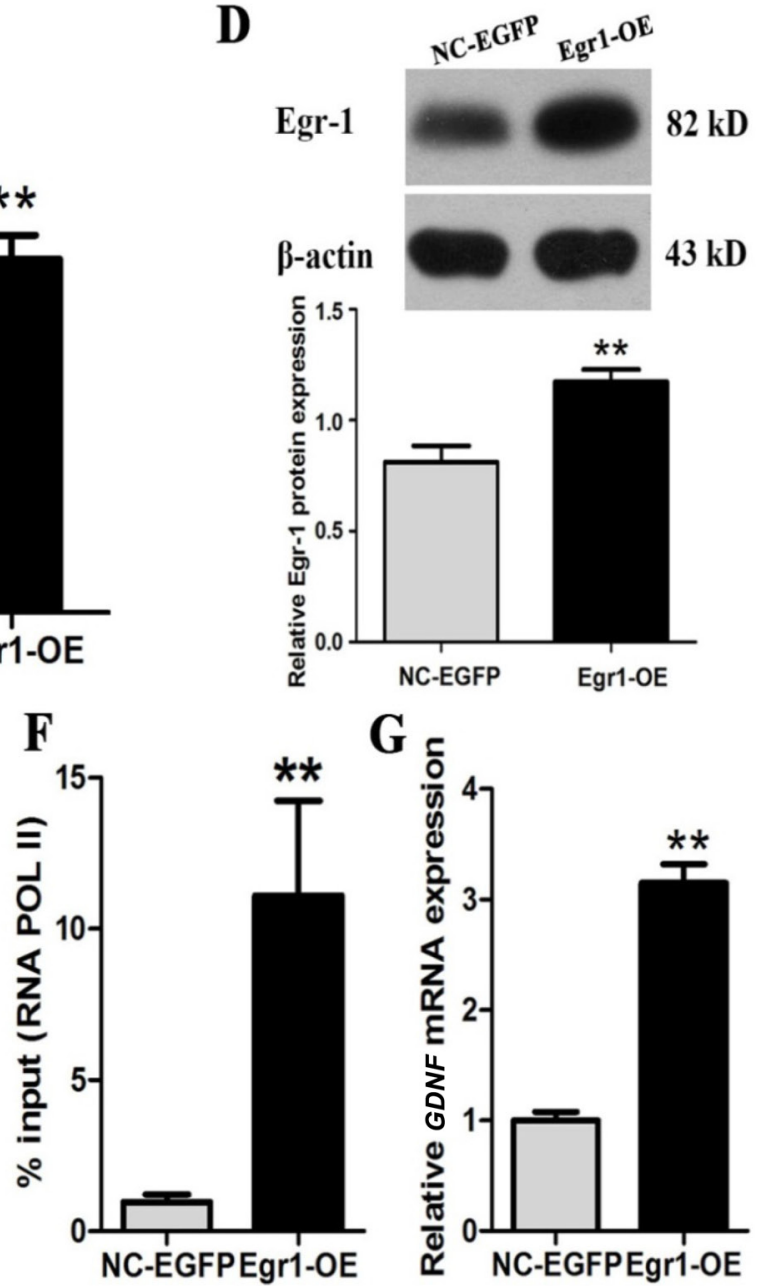

$\mathbf{G}$

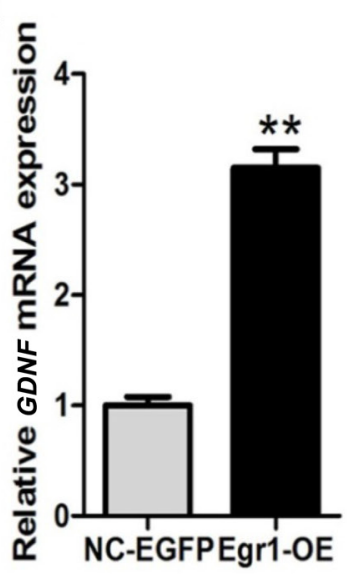

Figure 5: Egr-1 overexpression significantly increased Egr-1 binding to GDNF promoter II, RNA POL II recruitment, and $G D N F$ transcription in rat C6 glioma cells. C6 glioma cells in the logarithmic growth phase were inoculated into 6-well plates and then infected with $100 \mathrm{MOI}$ of lentivirus Egr1-OE and lentivirus NC-EGFP when the cells reached $70 \%$ confluence. After $72 \mathrm{~h}$, the virus infection rate was observed under the fluorescence microscope. (A, B) More than 90\% of C6 glioma cells had normal morphology and showed green fluorescence. Real-time PCR, western blot, and ChIP-PCR showed that (C) Egr-1 mRNA and (D) protein expression significantly increased in C6 cells infected with lentivirus Egr1-OE $(P<0.01)$. (E) Egr-1 binding to GDNF promoter II, (F) RNA POL II recruitment in that region, and $(\mathbf{G}) G D N F$ mRNA expression were also increased significantly $(P<0.05)$. The results are from three independent experiments. ${ }^{*} P<0.05, * * P<0.01$. The scale is $100 \mu \mathrm{m}$. 
GDNF promoter II. It has been pointed out that histone acetylation can affect transcription factor regulation [27]. Whether the regulation of GDNF induced by Egr1 overexpression depends on histone hyperacetylation in GDNF promoter II was tested in a C6 glioma cell model treated with curcumin as previously described [13]. The results showed that with histone hypoacetylation in the Egr-1 binding sites of GDNF promoter II, the binding of Egr-1 and RNA POL II to GDNF promoter II or $G D N F$ transcription could not be significantly increased, even with Egr-1 overexpression. This indicates that the regulation of GDNF induced by overexpressed Egr-1 and RNA POL II in high-grade glioma cells is dependent on histone hyperacetylation of GDNF promoter II.

How does Egr-1 interact with RNA POL II under histone hyperacetylation in GDNF promoter II? This needed to be further investigated, but it has been suggested that certain gene-specific transcription factors can promote RNA POL II recruitment [28, 29]. It was therefore speculated that Egr-1 might have a similar effect, which led us to examine Egr-1 binding to RNA POL II in C6 glioma cells. The results showed that they co-existed in glioma cell nuclei with local overlapping regions, but the proteins were not bound to each other. This suggests that Egr-1 may be involved in RNA POL II recruitment, but not by direct binding. Egr-1 has three typical zinc finger domains that can bind to the three base pairs in the DNA major groove in the gene promoter. This widens and deepens the DNA major groove, affecting the conformation of nearby DNA molecules [30, 31]. Indeed, our previous studies showed that both histone hyperacetylation [13] and $\mathrm{CpG}$ hypermethylation [9] occurred in the Egr-1 binding sites of GDNF promoter II in high-grade glioma cells. A recent study found that $\mathrm{CpG}$ hypermethylation in the Egr-1 binding motif did not affect its binding to Egr-1 [32]. However, such hypermethylation
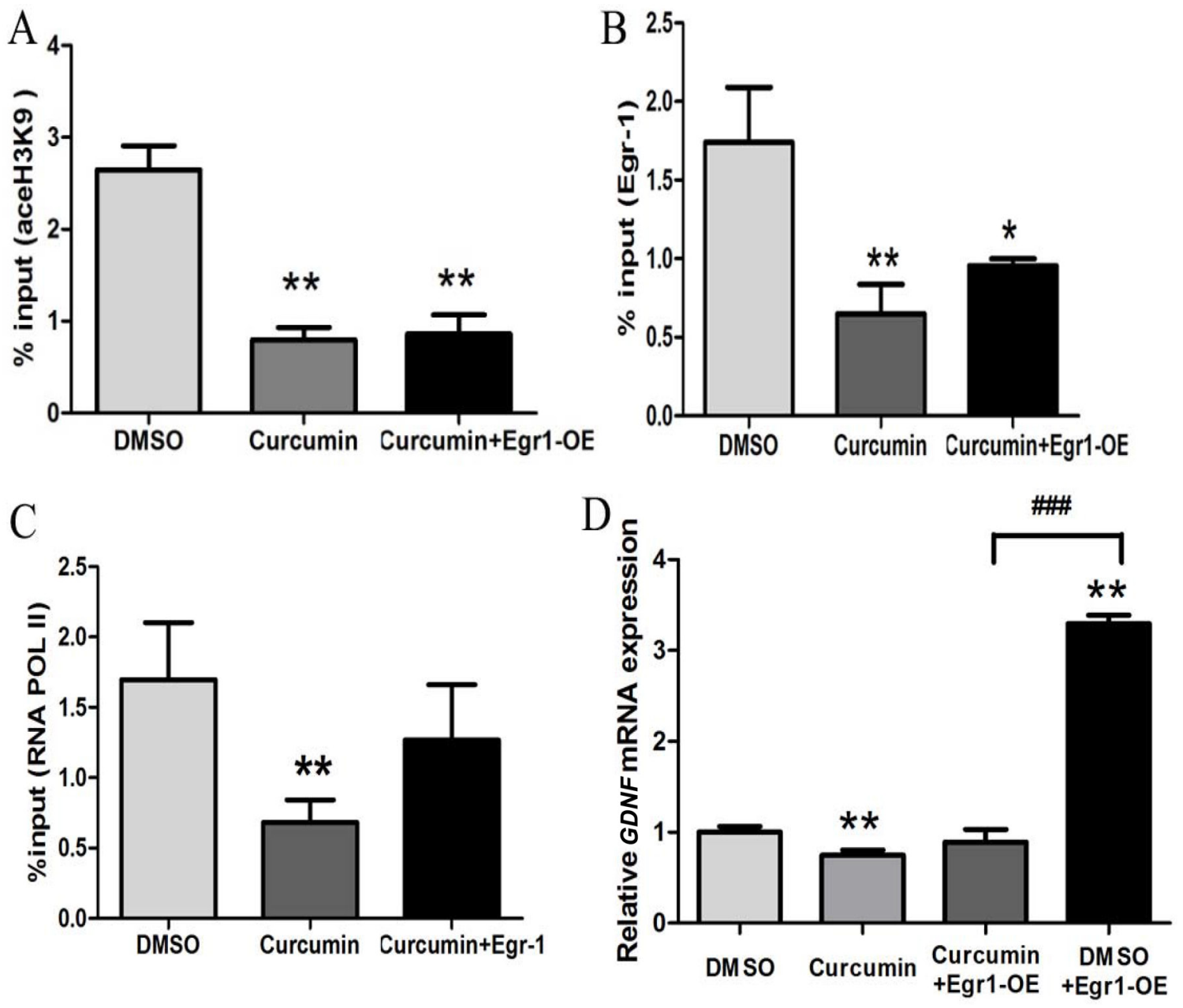

Figure 6: Histone hypoacetylation inhibited $G D N F$ transcription induced by Egr-1 overexpression in $G D N F$ promoter II in C6 glioma cells. Cells in the logarithmic growth phase were inoculated into 6-well plates and treated with $50 \mu \mathrm{M}$ curcumin or $0.25 \%$ DMSO for $24 \mathrm{~h}$ when the cells reached $50 \%$ confluence. The cells were then infected with $100 \mathrm{MOI}$ of lentivirus Egr 1-OE for $72 \mathrm{~h}$. (A) H3K9 acetylation of the Egr-1 binding sites of GDNF promoter II in C6 glioma cells was measured using ChIP-PCR. (B) Egr-1 binding was measured using ChIP-PCR. (C) RNA POL II recruitment in the Egr-1 binding sites of GDNF promoter II in C6 glioma cells was measured using ChIP-PCR. (D) Relative GDNF mRNA expression was measured by real-time PCR. ${ }^{*} P<0.05, * * P<0.01,{ }^{\# \#} P<0.001$. 
might inhibit RNA POL II recruitment [27, 33]. Therefore, it was hypothesized that Egr-1 might bind to the DNA major groove in the hypermethylated Egr-1 binding sites of GDNF promoter II via its zinc finger domains, deepening the DNA major groove and fully opening the DNA molecules. This would further promote RNA POL II recruitment in that region. Indeed, Egr-1 is able to influence the binding of other factors to the gene promoter by binding to the promoter [34].

In conclusion, we found that $\mathrm{H} 3 \mathrm{~K} 9$ acetylation in the Egr-1 binding sites of GDNF promoter II, binding of Egr-1 to such sites, RNA POL II recruitment in that region, and Egr-1 expression were significantly increased in highgrade glioma tissue. High Egr-1 expression promoted the binding of Egr-1 and RNA POL II to GDNF promoter II and enhanced GDNF transcription. Furthermore, studies of a C6 glioma cell model with curcumin-induced histone hypoacetylation in GDNF promoter II revealed that low acetylation in the Egr-1 binding sites of GDNF promoter II significantly decreased Egr-1 binding to GDNF promoter II, RNA POL II recruitment, and GDNF transcription in C6 glioma cells. These effects could not be reversed by Egr-1 overexpression. In addition, Egr1 and RNA POL II co-existed in C6 cells but were not bound to each other. Collectively, these findings indicate that in high-grade glioma cells, highly expressed Egr1 may be involved in recruiting RNA POL II in GDNF promoter II in a non-binding manner, suggesting that Egr-1 helps regulate high-level GDNF transcription. Furthermore, such regulation is dependent on histone hyperacetylation in GDNF promoter II. Our next study will focus on the how Egr-1 and RNA POL II jointly promote high-level GDNF transcription in high-grade glioma cells.

\section{MATERIALS AND METHODS}

\section{Tissue samples}

Tissue samples were derived from six patients with acute brain trauma given intracranial decompression and 12 human glioma tissue samples (World Health Organization [WHO] grades I-IV) acquired from the affiliated hospitals of Suzhou University were utilized for ChIP and Western blot assays. Biopsied glioma tissues derived from patients who had not yet undergone cancer therapy were randomly sampled. From these, the specimens with pathological grades I-II were assigned to the low-grade glioma group, while those with pathological grades III-IV were assigned to the high-grade glioma group.

\section{Cell culture}

Cryopreserved rat C6 astroglioma cell line and normal astrocytes were used for the experiments. Cell culture was performed as previously described [11], using medium composed of modified Eagle's medium (Gibco/ Invitrogen, Carlsbad, CA, USA) containing 10\% FBS (Gibco/Invitrogen), $100 \mathrm{U} / \mathrm{mL}$ penicillin, and $100 \mathrm{U} / \mathrm{mL}$ streptomycin. All cells were maintained in a $37^{\circ} \mathrm{C}$ incubator with a $5 \% \mathrm{CO}_{2}$ humidified atmosphere. Healthy cells derived after two or three passages were chosen for the following experiments.

\section{Chromatin immunoprecipitation-polymerase chain reaction (ChIP-PCR)}

Using the acetyl-histone H3 (Lys9) polyclonal antibody (cat. no.: 07-352, Millipore, Billerica, MA,

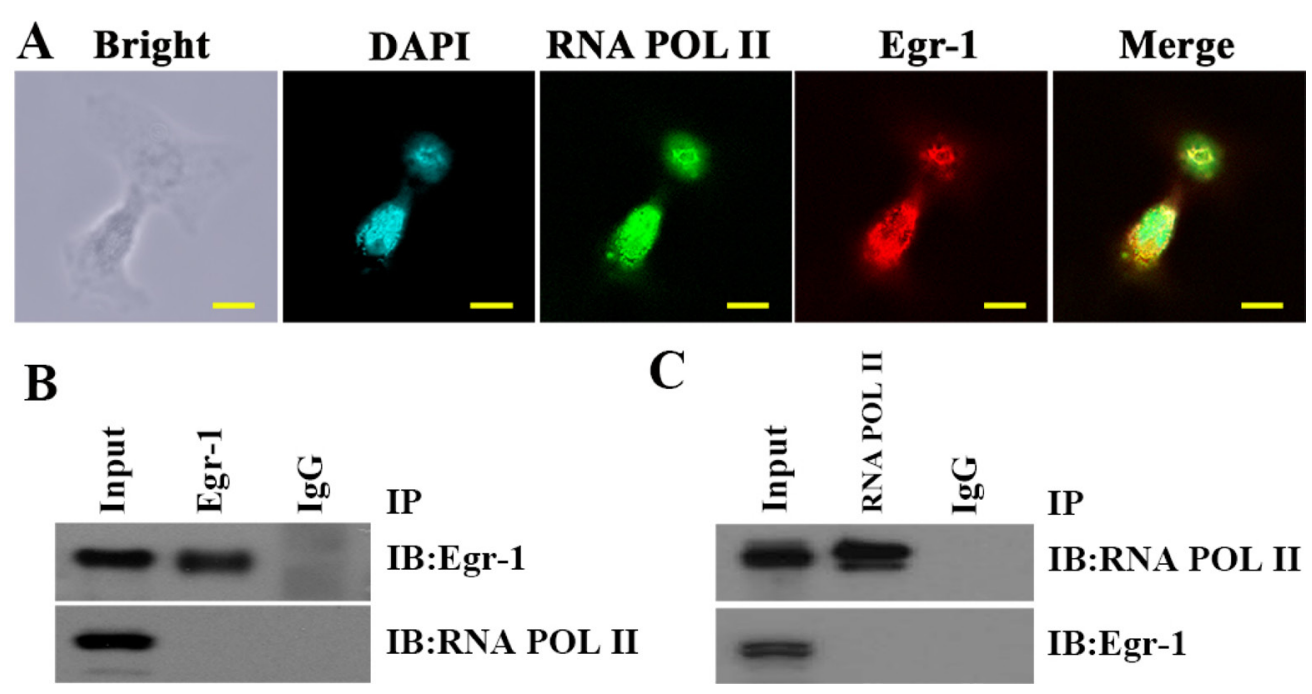

Figure 7: Co-existence but not binding of Egr-1 and RNA POL II in C6 glioma cell nuclei. (A) Egr-1 and RNA POL II protein locations in C6 glioma cells were determined by immunofluorescence. They were co-expressed in the nuclei of C6 cells with local overlapping regions. The scale is $100 \mu \mathrm{m}$. (B, C) The binding of Egr-1 to RNA POL II in C6 glioma cell nuclei was measured by co-IP with an Egr-1 antibody followed by western blotting with an RNA polymerase II antibody. Next, the reverse protocol was carried out. The results showed that the proteins were not bound in the nuclei of C6 glioma cells. 
USA), Egr-1 polyclonal antibody (cat. no.: sc-110X, Santa Cruz Biotechnology [SCBT], Santa Cruz, CA, USA), RNA POL II monoclonal antibody (cat. no.: 05-623, Millipore, Billerica, MA, USA) and normal IgG (cat. no.: sc-2027X, SCBT), ChIP was performed according to the the EZchip chromatin immunoprecipitation kit manufacturer's instructions (Millipore, Billerica, MA, USA). Routine polymerase chain reaction (PCR) and real-time PCR were used to detect DNA from immunoprecipitated target antibody. After conventional PCR, the PCR products were detected by agarose gel electrophoresis. After real-time PCR, data quantification was analyzed relative to gene expression data using the $2^{-\Delta \Delta \mathrm{Ct}}$ method, and the results were displayed as \% Input $=2$ (CTInput-CTChP) $\times$ Input dilution factor $\times 100$. The PCR primer was synthesized by Kangchen Bio-tech Inc. (Shanghai, China), and the sequence is shown in Table 1.

\section{Recombinant lentivirus construction and Infection}

The full-length rat $E g r-1$ cDNA fragment (NM_012551.2) was amplified by PCR technology. The PCR primers were synthesized as follows (Genechem Co., Ltd, Shanghai, China): Egr-1-AgeI-F 5'-ATGGACAACTACCCCAAACT-3', and Egr-1-AgeI-R 5'-TTAGCAAATTTCAATTGTCCTAGG-3'. The lentiviral vector Ubi-MCS-GFP (Genechem) was digested by the restriction enzyme AgeI. Then the $E g r-1$ cDNA fragments were ligated into the lentiviral vector Ubi-MCS-GFP. The primer (5'-AAGCGCTGCTGCCGCTGTTGCT-3') located in the coding sequence of the Egr-1 cDNA was used in PCR to identify positive transformants. Positive clones, as confirmed by PCR, were chosen for sequencing. Recombinant lentiviruses, which coexpress enhanced green fluorescent protein (EGFP) and Egr-1 sequence, were produced by $293 \mathrm{~T}$ cells following the cotransfection of Ubi-Egrl-EGFP and the packaging plasmids pHelper1.0 and pHelper2.0 (Genechem). The virus titer was detected by real-time PCR after concentrating and harvesting the viral supernatant. C6 astroglioma cells were plated at a density of $5 \times 10^{4}$ cells/well in a 6 -well plate (Corning, NY, USA). After $24 \mathrm{~h}$, cells were infected by $100 \mathrm{MOI}$ concentrated lentivirus in the presence of polybrene $(8 \mu \mathrm{g} / \mathrm{ml}) .72 \mathrm{~h}$ after infection, cells were harvested for the following experiments.

\section{RNA extraction and real-time PCR}

RNA extraction and real-time PCR were performed as previously described [13]. Glyceraldehyde-3-phosphate dehydrogenase (GAPDH) mRNA expression was used as the internal reference for every sample, and the relative mRNA expression levels of the target gene were calculated by relative quantification $\left(2^{-\Delta \Delta C T}\right)$. The forward and reverse primer sequences for the target gene amplification as well as internal reference gene are shown in Table 2.

\section{Western blot}

Total protein was extracted from each sample with NP-40 lysis buffer. Total extracts were separated on a $10 \%$ SDS-PAGE gel and transferred onto NC membrane (Millipore, USA). Blots were blocked using TBST solution containing $5 \%$ non-fat milk, and incubated in rabbit antimouse Egr-1 polyclonal antibody (1:200, Santa-Cruz, USA) or a mouse anti-avian $\beta$-Actin monoclonal antibody (sc-47778, Santa-Cruz, USA). After series of washings with TBST, the NC membrane was incubated in IR-Dye $800 \mathrm{cw}$ or $680 \mathrm{rd}$ labeled secondary antibodies $(1: 10,000$, LI-COR), and then scanned using a LI-COR Odyssey imaging system (LiCor, Lincoln, NE). $\beta$-actin was used as the internal reference protein.

\section{Immunoprecipitation and immunoblotting}

Equal amounts of nuclear protein extracts prepared from $\mathrm{C} 6$ cells were incubated with the mouse monoclonal RNA polymerase II antibody (05-623, Millipore, Billerica, MA, USA), rabbit polyclonal Egr-1 antibody (sc-110X, SCBT, Santa Cruz, CA, USA) or normal IgG (sc-2027X, SCBT) overnight at $4^{\circ} \mathrm{C}$. Then, the agaroseconjugated protein- $\mathrm{A} / \mathrm{G}$ beads (SCBT) were added into the immunocomplex and the mixture was incubated at $4^{\circ} \mathrm{C}$ for another $12 \mathrm{~h}$. After extensive washing with ice-cold WB/IP lysis buffer with protease inhibitors, the beads were mixed with SDS loading buffer and boiled. Immunoblotting were performed as Western Blot described above.

\section{Immunofluorescence assay}

The $\mathrm{C} 6$ cells were grown on coverslips in a 24-well plate, fixed with $4 \%$ paraformaldehyde (w/v) for $40 \mathrm{~min}$, and permeabilized with $0.5 \%(\mathrm{w} / \mathrm{v})$ Triton X-100 in PBS for $15 \mathrm{~min}$. The cells were then blocked for $30 \mathrm{~min}$ in PBS containing $10 \%$ fetal bovine serum (FBS) followed by overnight incubation with the primary antibodies against Egr-1 (1: 250, Santa-Cruz, USA) and RNA polymerase II (1: 300, Millipore, USA). After series of washings with PBS, cells were incubated for $2 \mathrm{~h}$ with secondary antibodies (Life, US) conjugated with fluorescein isothiocyanate or tetra methyl rhodamine isothiocyanate (TRITC) in dark moist environment. The coverslips were mounted with hydromount containing DAPI to stain the nuclei (KeyGEN BioTECH, China). The localization of Egr-1 and RNA polymerase II protein was examined using fluorescence confocal microscope (Leica Microsystems).

\section{Statistical analysis}

Statistical analyses were carried out using the SPSS 16.0 software package (SPSS Inc., Chicago, IL, USA) and expressed as mean \pm standard deviation (mean \pm SD). Independent sample $t$ test was used to test the mean value 
Table 1: Primer sequence for ChIP-PCR

\begin{tabular}{clcc}
\hline \multicolumn{1}{c}{ Gene name } & \multicolumn{1}{c}{ Primer sequence } & Tq ( ${ }^{\circ}$ C) & Primer length (bp) \\
\hline \multirow{2}{*}{ Hum $G D N F-\mathrm{P}_{(-98 /+56)}$} & F:5' CTGCTCGGACCTCGGCTT 3' & 60 & 154 \\
& R:5' GGCAAGAGTTCGCAATCCTG 3' & 60 & 71 \\
Hum GDNF-P & F:5' GGCAGCTCCTTTTCTCGC 3' & \\
Rat-GDNF-P & R:5' CTTCCCGCTCGGGTGTCT 3' & F:5' CGAGGAGGTGCAGAGTGAGG3' & 60 \\
\hline
\end{tabular}

Table 2: Primer sequence for real-time PCR

\begin{tabular}{|c|c|c|c|}
\hline Gene name & Primer sequence & $\operatorname{Tq}\left({ }^{\circ} \mathrm{C}\right)$ & Primer length (bp) \\
\hline Hum Egr-1 & $\begin{array}{l}\text { F:5'GGTCAGTGGCCTAGTGAGC3' } \\
\text { R:5'GTGCCGCTGAGTAAATGGGA3' }\end{array}$ & 59 & 149 \\
\hline Hum $G A P D H$ & $\begin{array}{l}\text { F:5'GAAGGTGAAGGTCGGAGTC3' } \\
\text { F:5'GAAGATGGTGATGGGATTTC3' }\end{array}$ & 59 & 226 \\
\hline Rat Egr-1 & $\begin{array}{l}\mathrm{F}: 5^{\prime} \text { CACCTGACCACAGAGTCCTT3' } \\
\text { R:5'CCAGTATAGGTGATGGGAGG3' }\end{array}$ & 59 & 110 \\
\hline Rat GDNF & $\begin{array}{l}\text { F:5' GACTTGGGTTTGGGCTACGA3' } \\
\text { R:5' TGGTAAACCAGGCTGTCGTC3' }\end{array}$ & 59 & 209 \\
\hline Rat $G A P D H$ & $\begin{array}{l}\text { F:5' TCCCTCAAGATTGTCAGCAA3' } \\
\text { R:5' AGATCCACAACGGATACATT3' }\end{array}$ & 59 & 308 \\
\hline
\end{tabular}

in 2 groups. One-way analysis of variance (ANOVA) was used to determine the significance of any differences between experimental groups. A value of $P<0.05$ was considered as significantly different.

\section{ACKNOWLEDGMENTS AND FUNDING}

This project was supported by the National Natural Science Foundation of China (Grant number: 81602464 and 81372698), Natural Science Foundation of Jiangsu (BK20130212), China and Jiangsu Postdoctoral Science Foundation funded project (2013M540466 and 1301068C), College Students Innovation and Entrepreneurship Training Program of Jiangsu Province (SIETP, 201610313001Z and 201610313001) and A Project Funded by the Priority Academic Program Development of Jiangsu Higher Education Institutions (PAPD).

\section{CONFLICTS OF INTEREST}

There are no conflicts of interest.

\section{REFERENCES}

1. Lin LF, Doherty DH, Lile JD, Bektesh S, Collins F. GDNF: a glial cell line-derived neurotrophic factor for midbrain dopaminergic neurons. Science. 1993; 260:1130-32.

2. Grimm L, Holinski-Feder E, Teodoridis J, Scheffer B, Schindelhauer D, Meitinger T, Ueffing M. Analysis of the human GDNF gene reveals an inducible promoter, three exons, a triplet repeat within the $3^{\prime}$-UTR and alternative splice products. Hum Mol Genet. 1998; 7:1873-86.
3. Baecker PA, Lee WH, Verity AN, Eglen RM, Johnson RM. Characterization of a promoter for the human glial cell linederived neurotrophic factor gene. Brain Res Mol Brain Res. 1999; 69:209-22.

4. Shabtay-Orbach A, Amit M, Binenbaum Y, Na'ara S, Gil Z. Paracrine regulation of glioma cells invasion by astrocytes is mediated by glial-derived neurotrophic factor. Int $\mathbf{J}$ Cancer. 2015; 137:1012-20.

5. Qu DW, Liu Y, Wang L, Xiong Y, Zhang CL, Gao DS. Glial cell line-derived neurotrophic factor promotes proliferation of neuroglioma cells by up-regulation of cyclins PCNA and Ki-67. Eur Rev Med Pharmacol Sci. 2015; 19:2070-75.

6. Ku MC, Wolf SA, Respondek D, Matyash V, Pohlmann A, Waiczies S, Waiczies H, Niendorf T, Synowitz M, Glass R, Kettenmann H. GDNF mediates glioblastoma-induced microglia attraction but not astrogliosis. Acta Neuropathol. 2013; 125:609-20.

7. Lu DY, Leung YM, Cheung CW, Chen YR, Wong KL. Glial cell line-derived neurotrophic factor induces cell migration and matrix metalloproteinase-13 expression in glioma cells. Biochem Pharmacol. 2010; 80:1201-09.

8. Ng WH, Wan GQ, Peng ZN, Too HP. Glial cell-line derived neurotrophic factor (GDNF) family of ligands confer chemoresistance in a ligand-specific fashion in malignant gliomas. J Clin Neurosci. 2009; 16:427-36.

9. Yu ZQ, Zhang BL, Ren QX, Wang JC, Yu RT, Qu DW, Liu ZH, Xiong Y, Gao DS. Changes in transcriptional factor binding capacity resulting from promoter region methylation induce aberrantly high GDNF expression in human glioma. Mol Neurobiol. 2013; 48:571-80. 
10. Wiesenhofer B, Stockhammer G, Kostron H, Maier H, Hinterhuber H, Humpel C. Glial cell line-derived neurotrophic factor (GDNF) and its receptor (GFR-alpha 1) are strongly expressed in human gliomas. Acta Neuropathol. 2000; 99:131-37.

11. Yu ZQ, Zhang BL, Ni HB, Liu ZH, Wang JC, Ren QX, Mo JB, Xiong Y, Yao RQ, Gao DS. Hyperacetylation of histone H3K9 involved in the promotion of abnormally high transcription of the gdnf gene in glioma cells. Mol Neurobiol. 2014; 50:914-22.

12. Kim Y, Kim SH, Kim YS, Lee YH, Ha K, Shin SY. Imipramine activates glial cell line-derived neurotrophic factor via early growth response gene 1 in astrocytes. Prog Neuropsychopharmacol Biol Psychiatry. 2011; 35:1026-32.

13. Zhang BL, Ni HB, Liu J, Lei Y, Li H, Xiong Y, Yao R, Yu ZQ, Gao DS. Egr-1 participates in abnormally high gdnf gene transcription mediated by histone hyperacetylation in glioma cells. Biochim Biophys Acta. 2014; 1839:1161-9.

14. Shan LN, Song YG, Su D, Liu YL, Shi XB, Lu SJ. Early Growth Response Protein-1 Involves in Transforming Growth factor- $\beta 1$ Induced Epithelial-Mesenchymal Transition and Inhibits Migration of Non-Small-Cell Lung Cancer Cells. Asian Pac J Cancer Prev. 2015; 16:4137-42.

15. Kim JH, Jeong IY, Lim Y, Lee YH, Shin SY. Estrogen receptor beta stimulates Egr-1 transcription via MEK1/Erk/Elk-1 cascade in C6 glioma cells. BMB Rep. 2011; 44:452-57.

16. Mittelbronn M, Harter P, Warth A, Lupescu A, Schilbach K, Vollmann H, Capper D, Goeppert B, Frei K, Bertalanffy H, Weller M, Meyermann R, Lang F, et al. EGR-1 is regulated by $\mathrm{N}$-methyl-D-aspartate-receptor stimulation and associated with patient survival in human high grade astrocytomas. Brain Pathol. 2009; 19:195-204.

17. Peng WX, Xiong EM, Ge L, Wan YY, Zhang CL, Du FY, $\mathrm{Xu}$ M, Bhat RA, Jin J, Gong AH. Egr-1 promotes hypoxiainduced autophagy to enhance chemo-resistance of hepatocellular carcinoma cells. Exp Cell Res. 2016; 340:62-70.

18. Eid MA, Kumar MV, Iczkowski KA, Bostwick DG, Tindall DJ. Expression of early growth response genes in human prostate cancer. Cancer Res. 1998; 58:2461-68.

19. Han ZX, Wang XX, Zhang SN, Wu JX, Qian HY, Wen YY, Tian H, Pei DS, Zheng JN. Downregulation of PAK5 inhibits glioma cell migration and invasion potentially through the PAK5-Egr1-MMP2 signaling pathway. Brain Tumor Pathol. 2014; 31:234-41.

20. Liu C, Yao J, Mercola D, Adamson E. The transcription factor EGR-1 directly transactivates the fibronectin gene and enhances attachment of human glioblastoma cell line U251. J Biol Chem. 2000; 275:20315-23.

21. Furukawa T, Tanese N. Assembly of partial TFIID complexes in mammalian cells reveals distinct activities associated with individual TATA box-binding proteinassociated factors. J Biol Chem. 2000; 275:29847-56.

22. Muta K, Obata Y, Oka S, Abe S, Minami K, Kitamura M, Endo D, Koji T, Nishino T. Curcumin ameliorates nephrosclerosis via suppression of histone acetylation independent of hypertension. Nephrol Dial Transplant. 2016; 31:1615-23.

23. Das C, Lucia MS, Hansen KC, Tyler JK. CBP/p300mediated acetylation of histone $\mathrm{H} 3$ on lysine 56 . Nature. 2009; 459:113-17.

24. Balasubramanyam K, Varier RA, Altaf M, Swaminathan V, Siddappa NB, Ranga U, Kundu TK. Curcumin, a novel p300/CREB-binding protein-specific inhibitor of acetyltransferase, represses the acetylation of histone/ nonhistone proteins and histone acetyltransferase-dependent chromatin transcription. J Biol Chem. 2004; 279:51163-71.

25. Obara Y, Nemoto W, Kohno S, Murata T, Kaneda N, Nakahata N. Basic fibroblast growth factor promotes glial cell-derived neurotrophic factor gene expression mediated by activation of ERK5 in rat C6 glioma cells. Cell Signal. 2011; 23:666-72.

26. Shin SY, Song H, Kim CG, Choi YK, Lee KS, Lee SJ, Lee HJ, Lim Y, Lee YH. Egr-1 is necessary for fibroblast growth factor-2-induced transcriptional activation of the glial cell line-derived neurotrophic factor in murine astrocytes. J Biol Chem. 2009; 284:30583-93.

27. Lee JY, Kong G. Roles and epigenetic regulation of epithelial-mesenchymal transition and its transcription factors in cancer initiation and progression. Cell Mol Life Sci. 2016; 73:4643-60.

28. Sun J, Rockowitz S, Chauss D, Wang P, Kantorow M, Zheng D, Cvekl A. Chromatin features, RNA polymerase II and the comparative expression of lens genes encoding crystallins, transcription factors, and autophagy mediators. Mol Vis. 2015; 21:955-73.

29. Sperling AS, Jeong KS, Kitada T, Grunstein M. Topoisomerase II binds nucleosome-free DNA and acts redundantly with topoisomerase I to enhance recruitment of RNA Pol II in budding yeast. Proc Natl Acad Sci USA. 2011; 108:12693-98.

30. Pavletich NP, Pabo CO. Zinc finger-DNA recognition: crystal structure of a Zif268-DNA complex at $2.1 \mathrm{~A}$. Science. 1991; 252:809-17.

31. Esadze A, Chen C, Zandarashvili L, Roy S, Pettitt BM, Iwahara J. Changes in conformational dynamics of basic side chains upon protein-DNA association. Nucleic Acids Res. 2016; 44:6961-70.

32. Zandarashvili L, White MA, Esadze A, Iwahara J. Structural impact of complete $\mathrm{CpG}$ methylation within target DNA on specific complex formation of the inducible transcription factor Egr-1. FEBS Lett. 2015; 589:1748-53.

33. Kellner WA, Bell JS, Vertino PM. GC skew defines distinct RNA polymerase pause sites in $\mathrm{CpG}$ island promoters. Genome Res. 2015; 25:1600-09.

34. Mouillet JF, Sonnenberg-Hirche C, Yan X, Sadovsky Y. p300 regulates the synergy of steroidogenic factor-1 and early growth response-1 in activating luteinizing hormonebeta subunit gene. J Biol Chem. 2004; 279:7832-39. 